\title{
The 3-dimensional q-deformed harmonic oscillator and magic numbers of alkali metal clusters
}

\author{
Dennis Bonatsos $\#$, N. Karoussos $\#$, P. P. Raychev ${ }^{\dagger}$, R. P. Roussev ${ }^{\dagger}$, P. A. Terziev ${ }^{\dagger}$ \\ \# Institute of Nuclear Physics, N.C.S.R. "Demokritos" \\ GR-15310 Aghia Paraskevi, Attiki, Greece \\ $\dagger$ Institute for Nuclear Research and Nuclear Energy, Bulgarian Academy of Sciences \\ 72 Tzarigrad Road, BG-1784 Sofia, Bulgaria
}

\begin{abstract}
Magic numbers predicted by a 3-dimensional $q$-deformed harmonic oscillator with $\mathrm{u}_{q}(3)$ $\supset \mathrm{so}_{q}(3)$ symmetry are compared to experimental data for alkali metal clusters, as well as to theoretical predictions of jellium models, Woods-Saxon and wine bottle potentials, and to the classification scheme using the $3 n+l$ pseudo quantum number. The 3 -dimensional $q$ deformed harmonic oscillator correctly predicts all experimentally observed magic numbers up to 1500 (which is the expected limit of validity for theories based on the filling of electronic shells), thus indicating that $\mathrm{u}_{q}(3)$, which is a nonlinear extension of the $\mathrm{u}(3)$ symmetry of the spherical (3-dimensional isotropic) harmonic oscillator, is a good candidate for being the symmetry of systems of alkali metal clusters.
\end{abstract}


Metal clusters have been recently the subject of many investigations (see [1, 2, 3] for relevant reviews). One of the first fascinating findings in their study was the appearance of magic numbers [4, 5, 6, 7, 8, 9, 10], analogous to but different from the magic numbers appearing in the shell structure of atomic nuclei [1]. This analogy led to the early description of metal clusters in terms of the Nilsson-Clemenger model [12], which is a simplified version of the Nilsson model [13, 14] of atomic nuclei, in which no spin-orbit interaction is included. Further theoretical investigations in terms of the jellium model [15, 16] demonstrated that the mean field potential in the case of simple metal clusters bears great similarities to the Woods-Saxon potential of atomic nuclei, with a slight modification of the "wine bottle" type [17]. The Woods-Saxon potential itself looks like a harmonic oscillator truncated at a certain energy value and flattened at the bottom. It should also be recalled that an early schematic explanation of the magic numbers of metallic clusters has been given in terms of a scheme intermediate between the level scheme of the 3-dimensional harmonic oscillator and the square well [1]. Again in this case the intermediate potential resembles a harmonic oscillator flattened at the bottom.

On the other hand, modified versions of harmonic oscillators [18, 19] have been recently investigated in the novel mathematical framework of quantum algebras [20], which are nonlinear generalizations of the usual Lie algebras. The spectra of $q$-deformed oscillators increase either less rapidly (for $q$ being a phase factor, i.e. $q=e^{i \tau}$ with $\tau$ being real) or more rapidly (for $q$ being real, i.e. $q=e^{\tau}$ with $\tau$ being real) in comparison to the equidistant spectrum of the usual harmonic oscillator [21], while the corresponding (WKBequivalent) potentials [22] resemble the harmonic oscillator potential, truncated at a certain energy (for $q$ being a phase factor) or not (for $q$ being real), the deformation inflicting an overall widening or narrowing of the potential, depending on the value of the deformation parameter $q$.

Very recently, a $q$-deformed version of the 3-dimensional harmonic oscillator has been constructed [23], taking advantage of the $\mathrm{u}_{q}(3) \supset \mathrm{so}_{q}(3)$ symmetry [24, 25, 26, 27, 28, 29, 30]. The spectrum of this 3-dimensional $q$-deformed harmonic oscillator has been found [23] to reproduce very well the spectrum of the modified harmonic oscillator introduced by Nilsson [13, 14], without the spin-orbit interaction term. Since the Nilsson model without the spin orbit term is essentially the Nilsson-Clemenger model used for the description of metallic clusters [12], it is worth examining if the 3-dimensional $q$-deformed harmonic oscillator can reproduce the magic numbers of simple metallic clusters. This is the subject of the present investigation. 
The space of the 3-dimensional $q$-deformed harmonic oscillator consists of the completely symmetric irreducible representations of the quantum algebra $\mathrm{u}_{q}(3)$. In this space a deformed angular momentum algebra, $\mathrm{SO}_{q}(3)$, can be defined [23]. The Hamiltonian of the 3-dimensional $q$-deformed harmonic oscillator is defined so that it satisfies the following requirements:

a) It is an $\mathrm{So}_{q}(3)$ scalar, i.e. the energy is simultaneously measurable with the $q$-deformed angular momentum related to the algebra $\mathrm{so}_{q}(3)$ and its $z$-projection.

b) It conserves the number of bosons, in terms of which the quantum algebras $\mathrm{u}_{q}(3)$ and $\mathrm{SO}_{q}(3)$ are realized.

c) In the limit $q \rightarrow 1$ it is in agreement with the Hamiltonian of the usual 3-dimensional harmonic oscillator.

It has been proved [23] that the Hamiltonian of the 3-dimensional $q$-deformed harmonic oscillator satisfying the above requirements takes the form

$$
H_{q}=\hbar \omega_{0}\left\{[N] q^{N+1}-\frac{q\left(q-q^{-1}\right)}{[2]} C_{q}^{(2)}\right\} \text {, }
$$

where $N$ is the number operator and $C_{q}^{(2)}$ is the second order Casimir operator of the algebra $\mathrm{SO}_{q}(3)$, while

$$
[x]=\frac{q^{x}-q^{-x}}{q-q^{-1}}
$$

is the definition of $q$-numbers and $q$-operators.

The energy eigenvalues of the 3-dimensional $q$-deformed harmonic oscillator are then 23

$$
E_{q}(n, l)=\hbar \omega_{0}\left\{[n] q^{n+1}-\frac{q\left(q-q^{-1}\right)}{[2]}[l][l+1]\right\},
$$

where $n$ is the number of vibrational quanta and $l$ is the eigenvalue of the angular momentum, obtaining the values $l=n, n-2, \ldots, 0$ or 1 .

In the limit of $q \rightarrow 1$ one obtains $\lim _{q \rightarrow 1} E_{q}(n, l)=\hbar \omega_{0} n$, which coincides with the classical result.

For small values of the deformation parameter $\tau$ (where $q=e^{\tau}$ ) one can expand eq. (3) in powers of $\tau$ obtaining 23

$$
\begin{gathered}
E_{q}(n, l)=\hbar \omega_{0} n-\hbar \omega_{0} \tau(l(l+1)-n(n+1)) \\
-\hbar \omega_{0} \tau^{2}\left(l(l+1)-\frac{1}{3} n(n+1)(2 n+1)\right)+\mathcal{O}\left(\tau^{3}\right) .
\end{gathered}
$$


The last expression to leading order bears great similarity to the modified harmonic oscillator suggested by Nilsson [13, 14] (with the spin-orbit term omitted)

$$
V=\frac{1}{2} \hbar \omega \rho^{2}-\hbar \omega \kappa^{\prime}\left(\mathbf{L}^{2}-<\mathbf{L}^{2}>_{N}\right), \quad \rho=r \sqrt{\frac{M \omega}{\hbar}},
$$

where

$$
<\mathbf{L}^{2}>_{N}=\frac{N(N+3)}{2}
$$

The energy eigenvalues of Nilsson's modified harmonic oscillator are 13, 14

$$
E_{n l}=\hbar \omega n-\hbar \omega \mu^{\prime}\left(l(l+1)-\frac{1}{2} n(n+3)\right) .
$$

It has been proved [23] that the spectrum of the 3-dimensional $q$-deformed harmonic oscillator closely reproduces the spectrum of the modified harmonic oscillator of Nilsson. In both cases the effect of the $l(l+1)$ term is to flatten the bottom of the harmonic oscillator potential, thus making it to resemble the Woods-Saxon potential.

The level scheme of the 3-dimensional $q$-deformed harmonic oscillator (for $\hbar \omega_{0}=1$ and $\tau=0.038$ ) is given in Table 1, up to a certain energy. Each level is characterized by the quantum numbers $n$ (number of vibrational quanta) and $l$ (angular momentum). Next to each level its energy, the number of particles it can accommodate (which is equal to $2(2 l+1))$ and the total number of particles up to and including this level are given. If the energy difference between two successive levels is larger than 0.39 , it is considered as a gap separating two successive shells and the energy difference is reported between the two levels. In this way magic numbers can be easily read in the table: they are the numbers appearing above the gaps, written in boldface characters.

The magic numbers provided by the 3-dimensional $q$-deformed harmonic oscillator in Table 1 are compared to available experimental data for Na clusters [4, 5, 6, 8, 9] in Table 2 (columns 2-6). The following comments apply:

i) Only magic numbers up to 1500 are reported, since it is known that filling of electronic shells is expected to occur only up to this limit 4 . For large clusters beyond this point it is known that magic numbers can be explained by the completion of icosahedral or cuboctahedral shells of atoms [4].

ii) Up to 600 particles there is consistency among the various experiments and between the experimental results in one hand and our findings in the other.

iii) Beyond 600 particles the predictions of the three experiments, which report magic numbers in this region, are quite different. However, the predictions of all three experiments 
are well accommodated by the present model. Magic numbers 694, 832, 1012 are supported by the findings of both Martin et al. [4] and Bréchignac et al. [9], magic numbers 1206, 1410 are in agreement with the experimental findings of Martin et al. [4], magic numbers 912, 1284 are supported by the findings of Bréchignac et al., while magic numbers 676, 1100, 1314, 1502 are in agreement with the experimental findings of Pedersen et al. [8].

In Table 2 the predictions of three simple theoretical models [11] (non-deformed 3dimensional harmonic oscillator (column 9), square well potential (column 8), rounded square well potential (intermediate between the previous two, column 7) ) are also reported for comparison. It is clear that the predictions of the non-deformed 3-dimensional harmonic oscillator are in agreement with the experimental data only up to magic number 40, while the other two models give correctly a few more magic numbers $(58,92,138)$, although they already fail by predicting magic numbers at $68,70,106,112,156$, which are not observed.

It should be noticed at this point that the first few magic numbers of alkali clusters (up to 92) can be correctly reproduced by the assumption of the formation of shells of atoms instead of shells of delocalized electrons [31], this assumption being applicable under conditions not favoring delocalization of the valence electrons of alkali atoms.

Comparisons among the present results, experimental data (by Martin et al. [4] (column 2), Pedersen et al. [8] (column 3) and Bréchignac et al. [9] (column 4) ) and theoretical predictions more sophisticated than these reported in Table 2, can be made in Table 3, where magic numbers predicted by various jellium model calculations (columns 5-8, 幽, 5, 2, 32]), Woods-Saxon and wine bottle potentials (column 9, [33]), as well as by a classification scheme using the $3 n+l$ pseudo quantum number (column 10, 四) are reported. The following observations can be made:

i) All magic numbers predicted by the 3-dimensional $q$-deformed harmonic oscillator are supported by at least one experiment, with no exception.

ii) Some of the jellium models, as well as the $3 n+l$ classification scheme, predict magic numbers at $186,540 / 542$, which are not supported by experiment. Some jellium models also predict a magic number at 748 or 758 , again without support from experiment. The WoodsSaxon and wine bottle potentials of Ref. [33] predict a magic number at 68 , for which no experimental support exists. The present scheme avoids problems at these numbers. It should be noticed, however, that in the cases of 186 and 542 the energy gap following them in the present scheme is 0.329 and 0.325 respectively (see Table 1), i.e. quite close to the threshold of 0.39 which we have considered as the minimum energy gap separating different 
shells. One could therefore qualitatively remark that 186 and 542 are "built in" the present scheme as "secondary" (not very pronounced) magic numbers.

The following general remarks can also be made:

i) It is quite remarkable that the 3-dimensional $q$-deformed harmonic oscillator reproduces the magic numbers at least as accurately as other, more sophisticated, models by using only one free parameter $\left(q=e^{\tau}\right)$. Once the parameter is fixed, the whole spectrum is fixed and no further manipulations can be made. This can be considered as evidence that the 3-dimensional $q$-deformed harmonic oscillator owns a symmetry (the $\mathrm{u}_{q}(3) \supset \operatorname{so}_{q}(3)$ symmetry) appropriate for the description of the physical systems under study.

ii) It has been remarked [4] that if $n$ is the number of nodes in the solution of the radial Schrödinger equation and $l$ is the angular momentum quantum number, then the degeneracy of energy levels of the hydrogen atom characterized by the same $n+l$ is due to the so(4) symmetry of this system, while the degeneracy of energy levels of the spherical harmonic oscillator (i.e. of the 3-dimensional isotropic harmonic oscillator) characterized by the same $2 n+l$ is due to the $\mathrm{su}(3)$ symmetry of this system. $3 n+l$ has been used [4] to approximate the magic numbers of alkali metal clusters with some success, but no relevant Lie symmetry could be determined (see also [34, 35]). In view of the present findings the lack of Lie symmetry related to $3 n+l$ is quite clear: the symmetry of the system appears to be a quantum algebraic symmetry $\left(\mathrm{u}_{q}(3)\right)$, which is a nonlinear extension of the Lie symmetry $\mathrm{u}(3)$.

iii) An interesting problem is to determine a WKB-equivalent potential giving (within this approximation) the same spectrum as the 3-dimensional $q$-deformed harmonic oscillator, using methods similar to these of Ref. [22]. The similarity between the results of the present model and these provided by the Woods-Saxon potential (column 9 in Table 3) suggests that the answer should be a harmonic oscillator potential flattened at the bottom, similar to the Woods-Saxon potential. If such a WKB-equivalent potential will show any similarity to a wine bottle shape, as several potentials used for the description of metal clusters do [15, 16, 17], remains to be seen.

In summary, we have shown that the 3-dimensional $q$-deformed harmonic oscillator with $\mathrm{u}_{q}(3) \supset \mathrm{so}_{q}(3)$ symmetry correctly predicts all experimentally observed magic numbers of alkali metal clusters up to 1500, which is the expected limit of validity for theories based on the filling of electronic shells. This indicates that $\mathrm{u}_{q}(3)$, which is a nonlinear deformation of the $\mathrm{u}(3)$ symmetry of the spherical (3-dimensional isotropic) harmonic oscillator, is a good candidate for being the symmetry of systems of alkali metal clusters. 
One of the authors (PPR) acknowledges support from the Bulgarian Ministry of Science and Education under contracts $\Phi-415$ and $\Phi-547$. 


\section{References}

[1] W. A. de Heer, Rev. Mod. Phys. 65 (1993) 611.

[2] M. Brack, Rev. Mod. Phys. 65 (1993) 677.

[3] V. O. Nesterenko, Fiz. Elem. Chastits At. Yadra 23 (1992) 1665 [Sov. J. Part. Nucl. 23 (1992) 726].

[4] T. P. Martin, T. Bergmann, H. Göhlich and T. Lange, Z. Phys. D 19 (1991) 25.

[5] S. Bjørnholm, J. Borggreen, O. Echt, K. Hansen, J. Pedersen and H. D. Rasmussen, Phys. Rev. Lett. 65 (1990) 1627.

[6] W. D. Knight, K. Clemenger, W. A. de Heer, W. A. Saunders, M. Y. Chou and M. L. Cohen, Phys. Rev. Lett. 52 (1984) 2141.

[7] W. D. Knight, W. A. de Heer, K. Clemenger and W. A. Saunders, Solid State Commun. $53(1985) 445$.

[8] J. Pedersen, S. Bjørnholm, J. Borggreen, K. Hansen, T. P. Martin and H. D. Rasmussen, Nature 353 (1991) 733.

[9] C. Bréchignac, Ph. Cahuzac, M. de Frutos, J.-Ph. Roux and K. Bowen, in Physics and Chemistry of Finite Systems: From Clusters to Crystals, ed. P. Jena et al. (Kluwer, Dordrecht, 1992) Vol. 1 p. 369.

[10] J. L. Persson, R. L. Whetten, H. P. Cheng and R. S. Berry, Chem. Phys. Lett. 186 (1991) 215.

[11] M. G. Mayer and J. H. D. Jensen, Elementary Theory of Nuclear Shell Structure (Wiley, New York, 1955).

[12] K. Clemenger, Phys. Rev. B 32 (1985) 1359.

[13] S. G. Nilsson, Mat. Fys. Medd. Dan Vid. Selsk. 29, no 16 (1955)

[14] S. G. Nilsson and I. Ragnarsson, Shapes and Shells in Nuclear Structure (Cambridge University Press, Cambridge, 1995).

[15] W. Ekardt, Phys. Rev. B 29 (1984) 1558. 
[16] D. E. Beck, Solid State Commun. 49 (1984) 381.

[17] B. A. Kotsos and M. E. Grypeos, in Atomic and Nuclear Clusters, ed. G. S. Anagnostatos and W. von Oertzen (Springer, Berlin, 1995) p. 242.

[18] L. C. Biedenharn, J. Phys. A 22 (1989) L873.

[19] A. J. Macfarlane, J. Phys. A 22 (1989) 4581.

[20] V. Chari and A. Pressley, A Guide to Quantum Groups (Cambridge University Press, Cambridge, 1994).

[21] D. Bonatsos, C. Daskaloyannis, P. Kolokotronis and D. Lenis, Rom. J. Phys. 41 (1996) 109.

[22] D. Bonatsos, C. Daskaloyannis and K. Kokkotas, J. Math. Phys. 33 (1992) 2958.

[23] P. P. Raychev, R. P. Roussev, N. Lo Iudice and P. A. Terziev, J. Phys. G 24 (1998) 1931.

[24] Yu. F. Smirnov, V. N. Tolstoy and Yu. I. Kharitonov, Sov. J. Nucl. Phys. 54 (1991) 437.

[25] Yu. F. Smirnov and Yu. I. Kharitonov, Phys. At. Nucl. 56 (1993) 1143.

[26] A. A. Malashin, Yu. F. Smirnov and Yu. I. Kharitonov, Phys. At. Nucl. 58 (1995) 595.

[27] A. A. Malashin, Yu. F. Smirnov and Yu. I. Kharitonov, Phys. At. Nucl. 58 (1995) 1031.

[28] J. Van der Jeugt, J. Phys. A 25 (1992) L213.

[29] J. Van der Jeugt, J. Math. Phys. 34 (1993) 1799.

[30] J. Van der Jeugt, Can. J. Phys. 72 (1994) 519.

[31] G. S. Anagnostatos, Phys. Lett. A 154 (1991) 169.

[32] A. Bulgac and C. Lewenkopf, Phys. Rev. Lett. 71 (1993) 4130.

[33] H. Nishioka, K. Hansen and B. R. Mottelson, Phys. Rev. B 42 (1990) 9377.

[34] E. Koch, Phys. Rev. A 54 (1996) 670.

[35] V. N. Ostrovsky, Phys. Rev. A 56 (1997) 626. 
Table 1: Energy spectrum, $E_{q}(n, l)$, of the 3-dimensional $q$-deformed harmonic oscillator (eq. (3)), for $\hbar \omega_{0}=1$ and $q=e^{\tau}$ with $\tau=0.038$. Each level is characterized by $n$ (the number of vibrational quanta) and $l$ (the angular momentum). $2(2 l+1)$ represents the number of particles each level can accommodate, while under "total" the total number of particles up to and including this level is given. Magic numbers, reported in boldface, correspond to energy gaps larger than 0.39 , reported between the relevant couples of energy levels. 
Table 1:

\begin{tabular}{|c|c|c|c|c|c|c|c|c|c|}
\hline$n$ & $l$ & $E_{q}(n, l)$ & $2(2 l+1)$ & total & $n$ & $l$ & $E_{q}(n, l)$ & $2(2 l+1)$ & total \\
\hline \multirow[t]{2}{*}{0} & 0 & 0.000 & 2 & 2 & 9 & 5 & 12.215 & 22 & 462 \\
\hline & & 1.000 & & & 11 & 11 & 12.315 & 46 & 508 \\
\hline \multirow[t]{2}{*}{1} & 1 & 1.000 & 6 & 8 & 10 & 8 & 12.614 & 34 & 542 \\
\hline & & 1.006 & & & 9 & 3 & 12.939 & 14 & 556 \\
\hline 2 & 2 & 2.006 & 10 & 18 & & & 0.397 & & \\
\hline \multirow[t]{2}{*}{2} & 0 & 2.243 & 2 & 20 & 9 & 1 & 13.336 & 6 & 562 \\
\hline & & 0.780 & & & 12 & 12 & 13.721 & 50 & 612 \\
\hline \multirow[t]{2}{*}{3} & 3 & 3.023 & 14 & 34 & 10 & 6 & 13.863 & 26 & 638 \\
\hline & & 0.397 & & & 11 & 9 & 14.154 & 38 & 676 \\
\hline \multirow[t]{2}{*}{3} & 1 & 3.420 & 6 & 40 & & & 0.603 & & \\
\hline & & 0.638 & & & 10 & 4 & 14.757 & 18 & 694 \\
\hline \multirow[t]{2}{*}{4} & 4 & 4.058 & 18 & 58 & & & 0.449 & & \\
\hline & & 0.559 & & & 13 & 13 & 15.206 & 54 & 748 \\
\hline 4 & 2 & 4.617 & 10 & 68 & 10 & 2 & 15.316 & 10 & 758 \\
\hline 4 & 0 & 4.854 & 2 & 70 & 10 & 0 & 15.554 & 2 & 760 \\
\hline \multirow[t]{2}{*}{5} & 5 & 5.116 & 22 & 92 & 11 & 7 & 15.592 & 30 & 790 \\
\hline & & 0.724 & & & 12 & 10 & 15.777 & 42 & 832 \\
\hline 5 & 3 & 5.841 & 14 & 106 & & & 0.884 & & \\
\hline 6 & 6 & 6.204 & 26 & 132 & 11 & 5 & 16.660 & 22 & 854 \\
\hline \multirow[t]{2}{*}{5} & 1 & 6.238 & 6 & 138 & 14 & 14 & 16.779 & 58 & 912 \\
\hline & & 0.860 & & & & & 0.606 & & \\
\hline 6 & 4 & 7.098 & 18 & 156 & 11 & 3 & 17.385 & 14 & 926 \\
\hline 7 & 7 & 7.328 & 30 & 186 & 12 & 8 & 17.410 & 34 & 960 \\
\hline 6 & 2 & 7.657 & 10 & 196 & 13 & 11 & 17.490 & 46 & 1006 \\
\hline \multirow[t]{2}{*}{6} & 0 & 7.895 & 2 & 198 & 11 & 1 & 17.782 & 6 & 1012 \\
\hline & & 0.502 & & & & & 0.667 & & \\
\hline 7 & 5 & 8.396 & 22 & 220 & 15 & 15 & 18.449 & 62 & 1074 \\
\hline \multirow[t]{2}{*}{8} & 8 & 8.494 & 34 & 254 & 12 & 6 & 18.660 & 26 & 1100 \\
\hline & & 0.627 & & & & & 0.645 & & \\
\hline \multirow[t]{2}{*}{7} & 3 & 9.121 & 14 & 268 & 14 & 12 & 19.305 & 50 & 1150 \\
\hline & & 0.397 & & & 13 & 9 & 19.330 & 38 & 1188 \\
\hline 7 & 1 & 9.518 & 6 & 274 & 12 & 4 & 19.554 & 18 & 1206 \\
\hline 9 & 9 & 9.709 & 38 & 312 & & & 0.559 & & \\
\hline \multirow[t]{2}{*}{8} & 6 & 9.743 & 26 & 338 & 12 & 2 & 20.113 & 10 & 1216 \\
\hline & & 0.894 & & & 16 & 16 & 20.226 & 66 & 1282 \\
\hline 8 & 4 & 10.637 & 18 & 356 & 12 & 0 & 20.350 & 2 & 1284 \\
\hline 10 & 10 & 10.980 & 42 & 398 & & & 0.417 & & \\
\hline 9 & 7 & 11.146 & 30 & 428 & 13 & 7 & 20.767 & 30 & 1314 \\
\hline 8 & 2 & 11.196 & 10 & 438 & & & 0.464 & & \\
\hline \multirow[t]{6}{*}{8} & 0 & 11.434 & 2 & 440 & 15 & 13 & 21.231 & 54 & 1368 \\
\hline & & 0.781 & & & 14 & 10 & $\begin{array}{r}21.360 \\
0.475\end{array}$ & 42 & 1410 \\
\hline & & & & & 13 & 5 & 21.835 & 22 & 1432 \\
\hline & & & & & 17 & 17 & 22.119 & 70 & 1502 \\
\hline & & & & & & & 0.441 & & \\
\hline & & & & & 13 & 3 & 22.560 & 14 & 1516 \\
\hline
\end{tabular}


Table 2: Magic numbers provided by the 3-dimensional $q$-deformed harmonic oscillator (Table 1), reported in column 1, are compared to the experimental data of Martin et al. [4] (column 2), Bjørnholm et al. [5] (column 3), Knight et al. [6] (column 4), Pedersen et al. [8] (column 5) and Bréchignac et al. [9] (column 6), concerning Na clusters. The magic numbers provided [11] by the (non-deformed) 3-dimensional harmonic oscillator (column 9), the square well potential (column 8) and a rounded square well potential intermediate between the previous two (column 7) are also shown for comparison. See text for discussion.

\begin{tabular}{|c|c|c|c|c|c|c|c|c|}
\hline present & $\begin{array}{c}\text { exp. } \\
\text { Ref. [4] }\end{array}$ & $\begin{array}{l}\text { exp. } \\
\text { Ref. [5] }\end{array}$ & $\begin{array}{c}\text { exp. } \\
\text { Ref. [6] }\end{array}$ & $\begin{array}{c}\text { exp. } \\
\text { Ref. [8] }\end{array}$ & $\begin{array}{c}\text { exp. } \\
\text { Ref. [9] }\end{array}$ & $\begin{array}{c}\text { int. } \\
\text { Ref. [11] }\end{array}$ & $\begin{array}{l}\text { sq. well } \\
\text { Ref. [11] }\end{array}$ & $\begin{array}{l}\text { h. osc. } \\
\text { Ref. [11] }\end{array}$ \\
\hline 2 & 2 & 2 & 2 & & & 2 & 2 & 2 \\
\hline 8 & 8 & 8 & 8 & & & 8 & 8 & 8 \\
\hline (18) & 18 & & & & & 18 & 18 & \\
\hline 20 & 20 & 20 & 20 & & & 20 & 20 & 20 \\
\hline 34 & 34 & & & & & 34 & 34 & \\
\hline 40 & 40 & 40 & 40 & 40 & & 40 & 40 & 40 \\
\hline \multirow[t]{2}{*}{58} & 58 & 58 & 58 & 58 & & 58 & 58 & \\
\hline & & & & & & 68,70 & 68 & 70 \\
\hline \multirow[t]{2}{*}{92} & 90,92 & 92 & 92 & 92 & 93 & 92 & 90,92 & \\
\hline & & & & & & 106,112 & 106 & 112 \\
\hline 138 & 138 & 138 & & 138 & 134 & 138 & 132,138 & \\
\hline 198 & $198 \pm 2$ & 196 & & 198 & 191 & 156 & 156 & 168 \\
\hline 254 & & $260 \pm 4$ & & & & & & \\
\hline 268 & $263 \pm 5$ & & & 264 & 262 & & & \\
\hline 338 & $341 \pm 5$ & $344 \pm 4$ & & 344 & 342 & & & \\
\hline 440 & $443 \pm 5$ & $440 \pm 2$ & & 442 & 442 & & & \\
\hline 556 & $557 \pm 5$ & $558 \pm 8$ & & 554 & 552 & & & \\
\hline 676 & & & & 680 & & & & \\
\hline 694 & $700 \pm 15$ & & & & 695 & & & \\
\hline 832 & $840 \pm 15$ & & & 800 & 822 & & & \\
\hline 912 & & & & & 902 & & & \\
\hline 1012 & $1040 \pm 20$ & & & 970 & 1025 & & & \\
\hline 1100 & & & & 1120 & & & & \\
\hline 1206 & $1220 \pm 20$ & & & & & & & \\
\hline 1284 & & & & & 1297 & & & \\
\hline 1314 & & & & 1310 & & & & \\
\hline 1410 & $1430 \pm 20$ & & & & & & & \\
\hline 1502 & & & & 1500 & & & & \\
\hline
\end{tabular}


Table 3: Magic numbers provided by the 3-dimensional $q$-deformed harmonic oscillator (Table 1), reported in column 1, are compared to the experimental data of Martin et al. [4] (column 2), Pedersen et al. [8] (column 3), and Bréchignac et al. [9] (column 4), as well as to the theoretical predictions of various jellium model calculations reported by Martin et al. [4] (column 5), Bjørnholm et al. [5] (column 6), Brack [2] (column 7), Bulgac and Lewenkopf [32] (column 8), the theoretical predictions of Woods-Saxon and wine bottle potentials reported by Nishioka et al. [33] (column 9), as well as to the magic numbers predicted by the classification scheme using the $3 n+l$ pseudo quantum number, reported by Martin et al. [4] (column 10). See text for discussion.

\begin{tabular}{|c|c|c|c|c|c|c|c|c|c|}
\hline present & $\begin{array}{c}\text { exp. } \\
\text { Ref.[4] }\end{array}$ & $\begin{array}{c}\text { exp. } \\
\text { Ref.[8] }\end{array}$ & $\begin{array}{c}\text { exp. } \\
\text { Ref.[9] }\end{array}$ & $\begin{array}{c}\text { jell. } \\
\text { Ref.[4] }\end{array}$ & $\begin{array}{c}\text { jell. } \\
\text { Ref.[5] }\end{array}$ & $\begin{array}{c}\text { jell. } \\
\text { Ref.[2] }\end{array}$ & $\begin{array}{c}\text { jell. } \\
\text { Ref.[32] }\end{array}$ & $\begin{array}{c}\text { WS } \\
\text { Ref.[33] }\end{array}$ & $\begin{array}{l}3 n+l \\
\text { Ref.[4] }\end{array}$ \\
\hline 2 & 2 & & & 2 & 2 & 2 & & 2 & 2 \\
\hline 8 & 8 & & & 8 & 8 & 8 & & 8 & 8 \\
\hline (18) & 18 & & & 18 & 18 & & & & 18 \\
\hline 20 & 20 & & & $(20)$ & 20 & 20 & & 20 & \\
\hline 34 & 34 & & & 34 & 34 & 34 & 34 & & 34 \\
\hline 40 & 40 & 40 & & $(40)$ & 40 & & & 40 & \\
\hline 58 & 58 & 58 & & 58 & 58 & 58 & 58 & $\begin{array}{l}58 \\
68\end{array}$ & 58 \\
\hline 92 & 90,92 & 92 & 93 & 92 & 92 & 92 & 92 & 92 & 90 \\
\hline 138 & 138 & 138 & 134 & $\begin{array}{l}134 \\
186\end{array}$ & $\begin{array}{l}138 \\
186\end{array}$ & $\begin{array}{l}138 \\
186\end{array}$ & $\begin{array}{l}138 \\
186\end{array}$ & 138 & $\begin{array}{l}132 \\
186\end{array}$ \\
\hline 198 & $198 \pm 2$ & 198 & 191 & (196) & 196 & & & 198 & \\
\hline $\begin{array}{l}254 \\
268\end{array}$ & $263 \pm 5$ & 264 & 262 & $\begin{array}{c}254 \\
(268)\end{array}$ & 254 & 254 & 254 & $\begin{array}{l}254 \\
268\end{array}$ & 252 \\
\hline 338 & $341 \pm 5$ & 344 & 342 & $338(356)$ & 338 & 338 & 338 & 338 & 332 \\
\hline 440 & $443 \pm 5$ & 442 & 442 & 440 & 440 & $\begin{array}{c}438,440 \\
542\end{array}$ & $\begin{array}{l}440 \\
542\end{array}$ & 440 & $\begin{array}{l}428 \\
540\end{array}$ \\
\hline $\begin{array}{l}556 \\
676\end{array}$ & $557 \pm 5$ & $\begin{array}{l}554 \\
680\end{array}$ & 552 & 562 & $\begin{array}{l}556 \\
676\end{array}$ & $\begin{array}{l}556 \\
676\end{array}$ & $\begin{array}{l}556 \\
676\end{array}$ & 562 & 670 \\
\hline 694 & $700 \pm 15$ & & 695 & 704 & & 758 & 748 & 694 & \\
\hline $\begin{array}{l}832 \\
912\end{array}$ & $840 \pm 15$ & 800 & $\begin{array}{l}822 \\
902\end{array}$ & 852 & 832 & $\begin{array}{l}832 \\
912\end{array}$ & $\begin{array}{l}832 \\
912\end{array}$ & 832 & 820 \\
\hline 1012 & $1040 \pm 20$ & 970 & 1025 & & & 1074 & 1074 & 1012 & 990 \\
\hline 1100 & & 1120 & & & & 1100 & 1100 & 1100 & \\
\hline 1206 & $1220 \pm 20$ & & & & & & & 1216 & 1182 \\
\hline $\begin{array}{l}1284 \\
1314\end{array}$ & & 1310 & 1297 & & & 1284 & 1284 & 1314 & \\
\hline 1410 & $1430 \pm 20$ & & & & & & & & 1398 \\
\hline 1502 & & 1500 & & & & 1502 & 1502 & 1516 & \\
\hline
\end{tabular}

